\author{
ОБ ИЗНОСЕ ОБОРУДОВАНИЯ В УСЛОВИЯХ ВИБРАЦИИ И \\ УДАРНЫХ НАГРУЗОК \\ (C) Илья Израилевич Блехман, Леонид Ильич Блехман, Владислав \\ Борисович Васильков, Кирилл Сергеевич Иванов, \\ Кира Саввовна Якимова \\ Федеральное государственное бюджетное учреждение науки Институт Проблем \\ Машиноведения Российской академии наук \\ blekhman@vibro.ipme.ru
}

\begin{abstract}
Аннотация. Показано что при действии вибрации и ударов номинально взаимно неподвижные детали, например, в болтовых и фланцевых соединениях, посадочных поверхностях подиипников качения, соединениях вал-втулка, листовых рессорах, шестернях, муфтах обнаруживают взаимную микро-, а иногда и макроподвижность $и$, как следствие, подвержены износу. На уровне простых физических моделей получены формуль для интенсивности износа, содержсащие эмпирический коэффициент. По физическому смыслу этот коэффициент представляет собой износ на единииу энергии, затрачиваемой при взаимном проскальзывании деталей. На основе полученных результатов даны некоторые рекомендации по конструированию и ремонтно-техническому обслужсиванию соответствующего оборудования.
\end{abstract}

Ключевые слова: вибрачия, ударные нагрузки, эксплуатация оборудования, трение, износ, фреттинг-износ, микропроскальзывание соединений, физические модели.

\title{
WEAR AND TEAR OF MACHINE PARTS AFFECTED BY VIBRATION AND PERCUSSIVE IMPACTS
}

(C) I.I. Blekhman, L.I. Blekhman, V.B. Vasilkov, K.S. Ivanov, K.S. Yakimova St. Petersburg, Institute for Problems in Mechanical Engineering RAS

Abstract. It is shown that nominally rigidly connected parts such as bolt connections, flange joints, roller bearing fit surfaces, bushing-shaft connections, leaf springs, gears, couplings when subjected to vibration and impact actions reveal micro- or macromobility between them involving their wear. Formulas containing an empirical coefficient have been derived from simple physical models to describe the wear intensity. In its physical sense this coefficient represents the wear per a unit of energy consumed on the parts mutual slip. Based on the results obtained, some recommendations are given with regard to designing and maintenance of equipment in question.

Key words: vibration, impact stress, exploitation, friction, wear and tear, fretting wear, microslip, physical models.

Многие машины для переработки природного и техногенного сырья, например, шаровые и стержневые мельницы, конусные и щековые дробилки, грохоты, конвейеры и питатели работают в условиях систематической вибрации и ударных нагрузок. То же относится к гидроэнергетическим агрегатам, строительным и другим машинам.

Известно, что вибрация и удары служат источником повышенной опасности разрушения деталей и даже катастроф. Снижение прочности материала при таких нагрузках - явление усталости - достаточно хорошо изучено. Этого нельзя сказать о другом важном эффекте - износе контактирующих номинально неподвижных деталей под действием указанных факторов, называемом фреттинг-износом. Такой износ имеет место в болтовых соединениях, посадочных поверхностях подшипников качения, соединениях вал-втулка, листовых рессорах, шестернях, муфтах и других устройствах. Продукты разрушения 
поверхностей при этом остаются в контактном зазоре и еще больше увеличивают износ. Кроме того, темп износа увеличивается из-за наличия воды и электрохимической коррозии.

Данный эффект лишь начинает изучаться (см., например, [1-4]).

Цель настоящей работы - подчеркнуть влияние этого эффекта, рассмотреть физическую причину данного вида износа, состоящую в возникновении относительной микро-, а иногда и макроподвижности сочленений, рассмотреть эффект на уровне простых физических моделей и дать некоторые рекомендации по конструированию и эксплуатации соответствующего оборудования.

Авторы благодарны Р.А. Арутюняну и Ю.А. Фадину за обсуждение и ценные замечания.

\section{1. Влияние вибрации и ударов на эффективные коэффициенты сухого трения. Микропроскальзывание контактирующих деталей.}

Под действием вибрации эффективные коэффициенты сухого трения покоя $f_{1}$, т.е. коэффициенты трения при действии постоянных или медленно изменяющихся сил, уменьшаются и могут обратиться в нуль, т.е. сухое трение как бы исчезает и «не держит» соответствующих нагрузок.

Пусть абсолютно твердое тело прижато к шероховатой плоскости силой $N$ и на него действует также гармоническая сила $\Phi=\Phi_{0} \sin \omega t$, которая может быть направлена как вдоль плоскости, так и в перпендикулярном и в поперечном направлениях (рис. $1, \mathrm{a})$.

a)

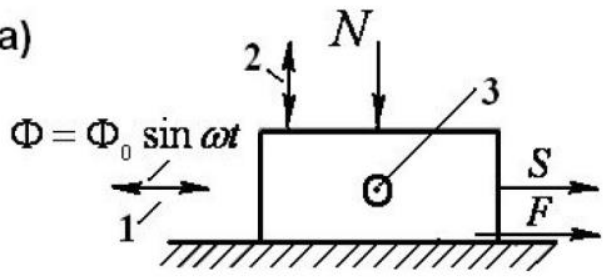

б)

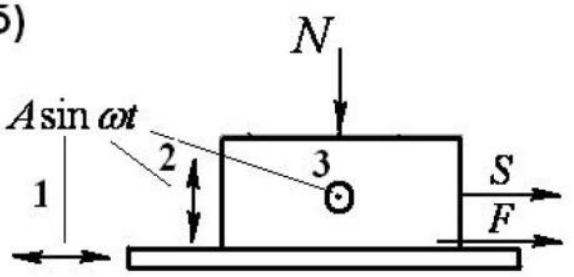

Рис. 1. К определению эффективных коэффициентов трения:

а) - случай действия вынуждающей силы:

1 - продольная сила, 2 - перпендикулярная, 3 - поперечная;

б) - случай вибрации плоскости:

1 - продольная вибрация, 2 - перпендикулярная, 3 - поперечная

Тогда эффективные коэффициенты трения покоя, т.е. коэффициенты трения по отношению к двигающей тело силе $S$ будут соответственно иметь значения $[5,6]$

$$
f_{1}^{(=)}=f_{1}\left(1-\frac{w}{f_{1}}\right), \quad f_{1}^{(\perp)}=f_{1}(1-w), \quad f_{1}^{(\bullet)}=f_{1} \sqrt{1-\left(w / f_{1}\right)^{2}},
$$

где $f_{1}-$ «обычный» коэффициент трения покоя, а

$$
w=\Phi_{0} / N
$$

- так называемый коэффициент перегрузки.

Формулы (1) остаются справедливыми в случае, когда сила Ф отсутствует (рис.1,6), но плоскость совершает гармонические колебания в соответствующих направлениях по закону $A \sin \omega t$ ( $A$-амплитуда, $\omega-$ частота колебаний). При этом следует лишь вычислять коэффициент перегрузки по формуле

$$
w=m A \omega^{2} / N,
$$

где $m$ - масса тела. Наконец, если нормальная сила $N$ представляет вес тела $m g$, то

$$
w=A \omega^{2} / g .
$$

Формулы (1) справедливы лишь до тех пор, пока коэффициенты $f_{1}^{(=)}, f_{1}^{(\perp)}$ и $f_{1}^{(\bullet)}$ положительны; при больших значениях параметра перегрузки $\quad w$ происходит уже кажущееся 
изменение характера трения; в этом случае можно считать, что эффективные коэффициенты трения равны нулю.

Приведенные формулы подтверждаются экспериментальными данными. Они объясняют эффект микропроскальзывания под действием вибрации номинально взаимно неподвижных деталей, контактирующих посредством сил сухого трения. Подробно этот эффект будет рассмотрен ниже на двух простых моделях.

Что касается действия ударных нагрузок, то, как показали, например, опыты Д.М. Толстого [7, 8], даже сравнительно слабое ударное воздействие может на некоторое, пусть весьма короткое время, привести к значительному снижению эффективного коэффициента трения покоя $f_{1}^{(\perp)}$. В упомянутых опытах при падении шарика с массой всего 0.45 г на тело («ползун») с массой 1176 г с высоты 4 см коэффициент $f_{1}^{(\perp)}$ уменьшался по сравнению с $f_{1}$ на $25 \%$. Теоретическое объяснение этого эффекта дано в работах $[6,9]$.

\section{2. Модель 1 - твердое тело на вибрирующей поверхности.}

Рассмотрим простейшую модель действия вибрации и ударов на контактирующие детали, взаимодействующие посредством сил сухого трения (рис. 2,a).

a)

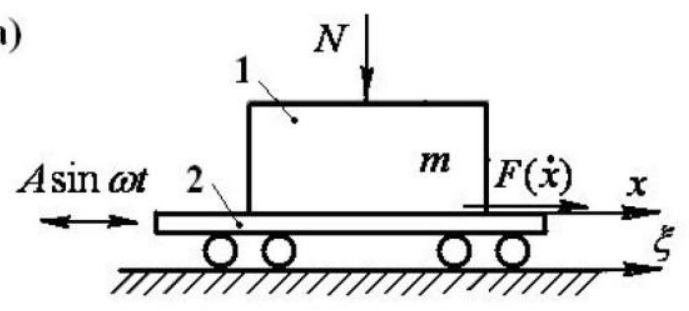

б)

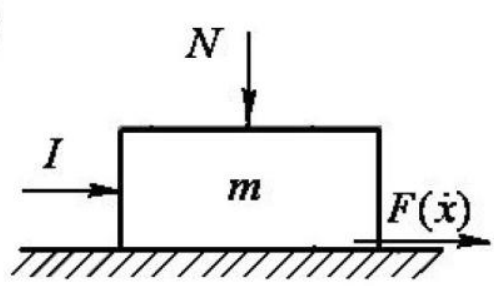

Рис. 2. Твердое тело на платформе:

а) - случай вибрации платформы, б) - случай действия удара

Твердое тело 1 некоторой массы $m$ расположено на жесткой платформе 2 , совершающей продольные колебания по закону

$$
\xi=A \sin \omega t,
$$

где $\xi$ - абсолютная координата платформы, $A$-амплитуда, $\omega$ - частота колебаний. Между телом и платформой действует сила сухого трения $F$; тело прижато к платформе некоторой постоянной силой $N$, которая может включать вес тела $m g$. Движение тела относительно платформы, определяемое посредством связанной с нею координаты $x$, описывается уравнением

$$
m \ddot{x}=m A \omega^{2} \sin \omega t+F(\dot{x}) .
$$

Здесь

$$
\begin{gathered}
F(\dot{x})=\left\{\begin{array}{rrr}
-f N & \text { при } & \dot{x}>0 \\
f N & \text { при } & \dot{x}<0
\end{array}\right. \\
-N f_{1}<F(\dot{x})<N f_{1} \text { при } \dot{x}=0,
\end{gathered}
$$

где $f$ и $f_{1}$ - коэффициенты сухого трения скольжения и покоя соответственно.

Устойчивые периодические движения, определяемые уравнением (6), подробно рассматривались в работах $[5,6,10]$. Приведем результаты этого решения в несколько иной форме.

Характер устанавливающихся движений зависит от двух безразмерных параметров

$$
w=\frac{m A \omega^{2}}{N f}, \quad w_{1}=\frac{m A \omega^{2}}{N f_{1}} .
$$


Если принять $f=0.7 f_{1}$, что примерно соответствует реальному соотношению между этими коэффициентами для многих материалов, то число параметров сводится к одному $w=m A \omega^{2} / N$. На рис. 3 представлена зависимость полуразмаха колебаний тела относительно платформы от этого параметра. При $w<1 / 0.7=1.43$ тело движется вместе с платформой проскальзывание отсутствует (область I). При $\frac{1}{0.7}=1.43<w<\frac{1}{0.472}=2.12$ тело скользит попеременно вперед и назад, останавливаясь при перемене направления скольжения на конечные промежутки времени (область II), а при $w>1 / 0.472=2.12$ (область III) - скользит, мгновенно изменяя направление скольжения.

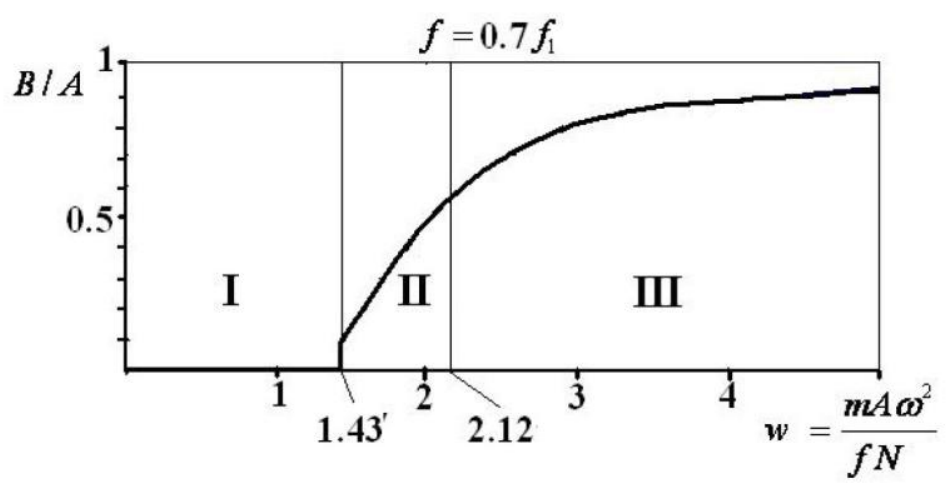

Рис. 3. Зависимость полуразмаха колебаний тела от параметра перегрузки

Полуразмах $B$ относительных колебаний тела при скольжении увеличивается при увеличении $w$, асимптотически приближаясь при $w \rightarrow \infty$ к величине амплитуды колебаний $A$.

Условием отсутствия проскальзывания тела, таким образом, является неравенство

$$
f_{1} N>m A \omega^{2}
$$

Или, при $f=0.7 f_{1}-$ неравенство

$$
f N>0.7 m A \omega^{2} .
$$

В случае удара по телу массой $m$ с импульсом $I$ (рис. 2,б) оно приобретает скорость $v=I / m$. В результате тело смещается на расстояние $B_{1}$, определяемое из равенства $\frac{1}{2} m v^{2}=f N B_{1}$, откуда

$$
B_{1}=\frac{m v^{2}}{2 f N}=\frac{I^{2}}{2 f N m} .
$$

Как следует из приведенных соотношений, если в случае действия вибрации можно исключить взаимное микропроскальзывание деталей путем обеспечения достаточно большого натяга $N$, то в случае ударных нагрузок гарантировать отсутствие микропроскальзывания нельзя даже при наличии весьма значительного натяга.

\section{3. Модель 2 - твердое тело с внутренней степенью свободы - влияние резонансных эффектов.}

Рассмотрим систему, отличающуюся от модели 1 тем, что внутри тела, масса которого $m_{1}$, размещено другое тело, имеющее массу $m_{2}$ (рис. 4,a). Это последнее тело связано с первым посредством упругого и демпфирующего элементов, жесткость и коэффициент демпфирования которых соответственно $c$ и $\beta$. Тело $m_{1}$, как и ранее, лежит на платформе, колеблющейся по закону (5); между телом $m_{1}$ и платформой действуют сила сухого трения $F(\dot{x})$. Тело $m_{2}$ может перемещаться относительно основного тела $m_{1}$ вдоль направления, параллельного плоскости контакта. 

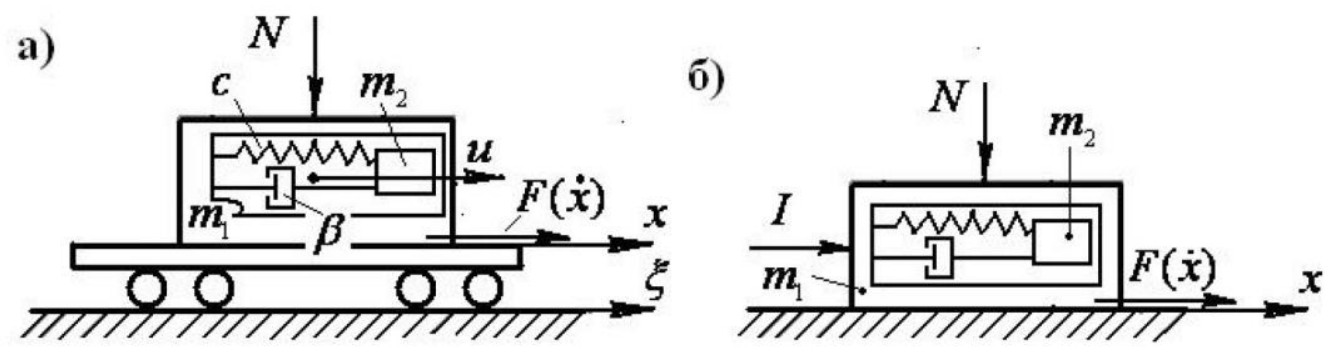

Рис. 4. Твердое тело с внутренней степенью свободы:

а) случай вибрации платформы, б) случай действия удара

Положение тела $m_{1}$ относительно платформы по-прежнему определено координатой $x$, а положение тела $m_{2}$ относительно тела $m_{1}$ - координатой $u$; последняя отсчитывается от положения, соответствующего недеформированному упругому элементу.

Уравнения движения описанной системы имеют вид

$$
\begin{aligned}
& m_{1} \ddot{x}=m_{1} A \omega^{2} \sin \omega t+c u+\beta \dot{u}+F(\dot{x}), \\
& m_{2} \ddot{u}=m_{2}\left(A \omega^{2} \sin \omega t-\ddot{x}\right)-c u-\beta \dot{u},
\end{aligned}
$$

где, как и ранее, сила трения $F(\dot{x})$ определяется соотношениями (7).

Точное аналитическое решение нелинейной системы (12)-(13) представляет существенные трудности. Приближенное решение более общей системы было получено в работе [11], задачей которой было исследование вибрационного перемещения системы. Здесь мы будем интересоваться периодическими колебательными режимами движения и приведем решения в несколько иных предположениях. А именно, примем в первом приближении, что на движение тела $m_{2}$ мало влияет движение основного тела $m_{1}$. Тогда в уравнении (13) можно пренебречь величиной $m_{2} \ddot{x}$ по сравнению с прочими и это уравнение примет вид

$$
m_{2} \ddot{u}+\beta \dot{u}+c u=m_{2} A \omega^{2} \sin \omega t .
$$

Решением этого уравнения, соответствующим установившимся вынужденным колебаниям, является

$$
u=A k \sin (\omega t+\alpha),
$$

где

$$
\begin{aligned}
& k=\frac{\omega^{2}}{\sqrt{\left(\lambda^{2}-\omega^{2}\right)^{2}+4 n^{2} \omega^{2}}}, \\
& \frac{c}{m_{2}}=\lambda^{2}, \quad \frac{\beta}{m_{2}}=2 n, \quad \sin \alpha=-2 \frac{n}{\omega} k, \quad \cos \alpha=\frac{\lambda^{2}-\omega^{2}}{\omega^{2}} k .
\end{aligned}
$$

В результате уравнение (12) примет вид

$$
m_{1} \ddot{x}=m_{1} A \omega^{2} \sin \omega t+c A k \sin (\omega t+\alpha)+\beta \omega A k \cos (\omega t+\alpha)+F(\dot{x}) .
$$

Это уравнение можно представить в виде

где

$$
m_{1} \ddot{x}=m_{1} A_{1} \omega^{2} \sin (\omega t+\varepsilon)+F(\dot{x}),
$$

$$
A_{1}=A \sqrt{1+2 k\left(\frac{\lambda_{1}^{2}}{\omega^{2}} \cos \alpha-2 \frac{n_{1}}{\omega} \sin \alpha\right)+k^{2}\left(\frac{\lambda_{1}^{4}}{\omega^{4}}+4\left(\frac{n_{1}}{\omega}\right)^{2}\right)}
$$

$\lambda_{1}^{2}=\frac{c}{m_{1}}, \quad 2 n_{1}=\frac{\beta}{m_{1}}$, а $\varepsilon-$ некоторая несущественная далее постоянная, которая может всегда быть обращена в нуль выбором начала отсчета времени $t$. Уравнение (17) совпадает с уравнением (6), полученным для одномассовой системы, и можно воспользоваться приведенным ранее решением. А именно, в предположении, что $f=0.7 f_{1}$ для нахождения 
полуразмаха колебаний $B=B_{2}$ будет справедлив график, представленный на рис. 3 с той разницей, что под амплитудой колебаний $A$ надо будет понимать амплитуду $A_{1}$, определяемую по формуле (18).

При желании найденным решением можно воспользоваться для вычисления следующего приближения. Однако в этом нет особой надобности, поскольку для уточнения решения можно воспользоваться программой, составленной для решения системы уравнений (12), (13). Вычисления по этой программе показывают, что найденное приближенное решение обладает удовлетворительной точностью.
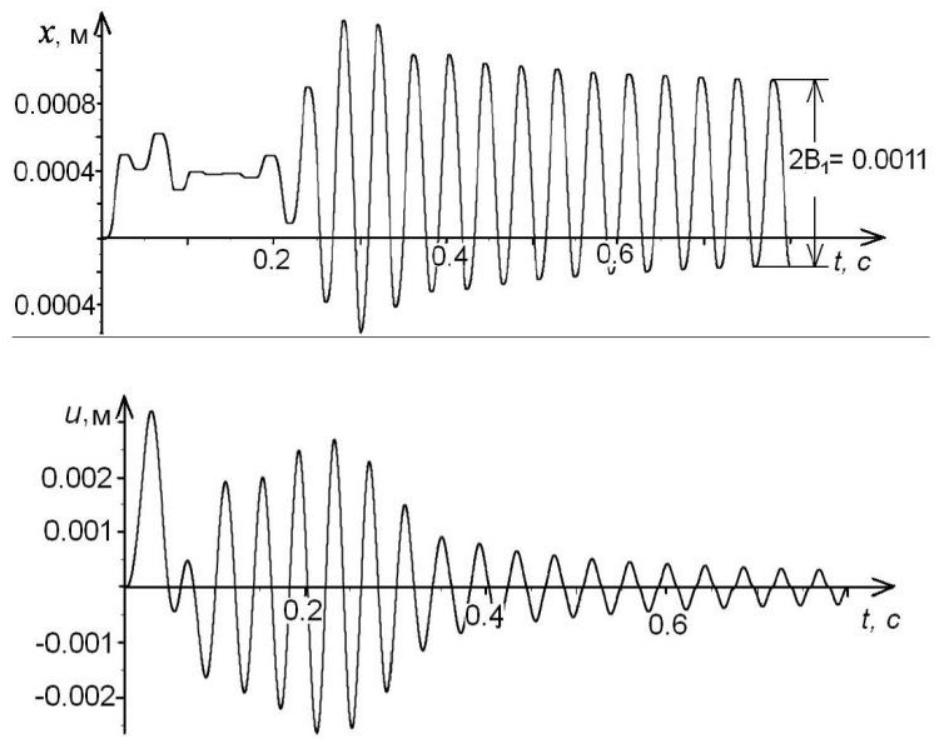

Рис. 5. Графики изменения координат $x$ и $u$ со временем

(значения параметров: $A=0.001 \mathrm{м} ; \omega=150 \mathrm{c}^{-1} ; c=9 \cdot 10^{4} \mathrm{H} / \mathrm{m} ; \beta=30 \mathrm{H} \cdot \mathrm{c} / \mathrm{m} ; \lambda_{2}=300 \mathrm{c}^{-1} ; f=0.5$;

$$
\left.m_{1}=3 \text { кг; } m_{2}=1 \text { кг; } N=100 \mathrm{H}\right)
$$

На рис. 5 в качестве примера представлены графики изменения со временем координат $x$ и $u$, а на рис. 6 - зависимость полуразмаха от частоты вибрации $\omega$. Как видно, наличие внутренней степени свободы может существенно усилить влияние вибрации на величину взаимного проскальзывания тела $m_{1}$ по основанию, если частота вибрации $\omega$ близка к частоте свободных колебаний $\lambda_{2}=\sqrt{c / m_{2}}$ тела $m_{2}$ внутри тела $m_{1}$. К такому же выводу приводят расчеты по формуле (18) и использование графика рис. 3.

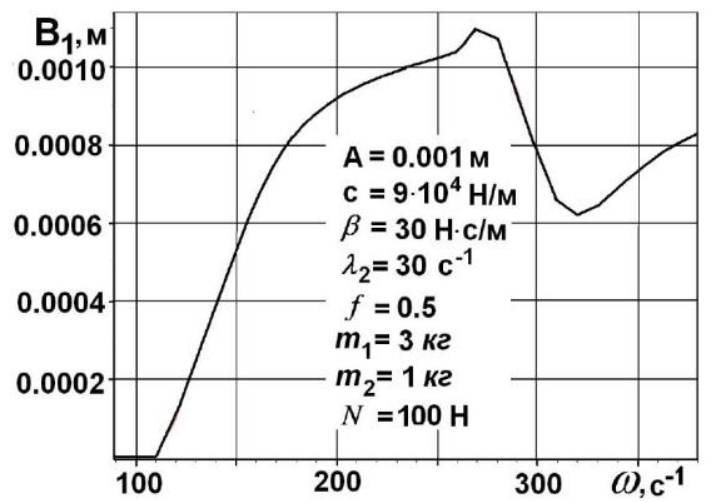

Рис. 6. Зависимость амплитуды колебаний тела $m_{1}$ от частоты вынужденных колебаний $\omega$ 
Для вычислений смещения тела $m_{1}$ при ударном воздействии остается справедливой формула (11), полученная для модели 1.

Как и в случае модели 1 , при вибрационном воздействии можно добиться устранения проскальзывания массы $m_{1}$ путем обеспечения достаточно большой величины силы прижатия $N$. При ударном же воздействии гарантировать отсутствие возникновения микропроскальзывания таким способом невозможно.

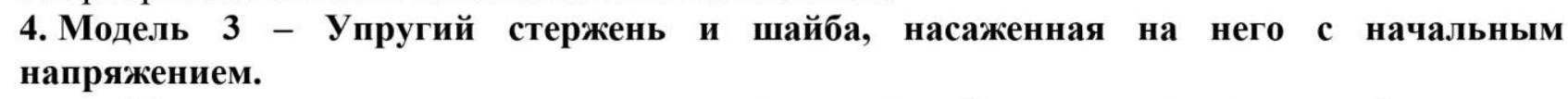
4. Модель 3 - Упругий стержень и шайба, насаженная на него с начальным напряжением.

Посадка с натягом - довольно распростарненный тип соединения частей машин и механизмов. Поведение частей таких соединений, состоящих из стержня и плотно насаженной на него шайбы, можно рассматривать, поираясь на модель частного случая так называемого маятника челомея. В работе [15] было показано, что в подобных системах при воздействии ударов и вибрации может возникать не только микропроскальзывание, но и вибрационное перемещение.

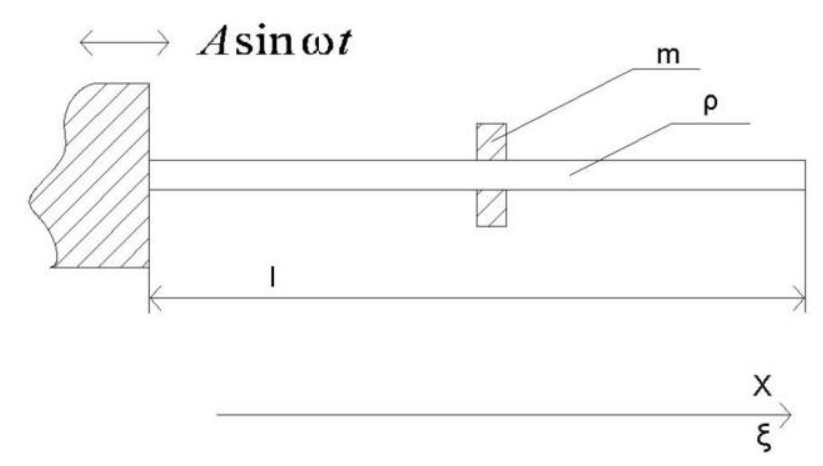

Рис. 7. Общая схема модели

Рассматриваемая система состоит из упругого стержня длины $l$ и жесткой шайбы массой $m$, насаженной на стержень с натягом с начальной деформацией $\varepsilon^{0}$ Основание стержня совершает поступательные колебания, которые могут быть записаны в неподвижной системе координат $\xi$ :

$$
\xi(t)=A \sin \omega t
$$

Уравнение движение шайбы на стержне можно записать в подвиженой системе координат в следующем виде:

$$
\begin{aligned}
& m \ddot{x}=m A \omega^{2} \sin \omega t+F_{f}(t, x, \dot{x}) \\
& F_{f}=\left\{\begin{array}{l}
-f N(t, x), \quad \dot{x}>0 \\
-m A \omega^{2} \sin \omega t, \dot{x} \equiv 0 \\
f N(t, x), \quad \dot{x}<0
\end{array}\right.
\end{aligned}
$$

где $x$ - координата сечения стержня в подвижной системе отсчета, связанной с колеблющимся основанием, и, в то же время, координата шайбы, находящейся над этим сечением, $F_{i}(t)$ - сила инерции в относительном движении, $F_{f}$ - сила трения, которая возникает между шайбой и стержнем, $m$ - масса шайбы, $A$ - амплитуда колебаний основания стержня, $\omega-$ частота

этих колебаний, $f$ - коэффициент трения, $N(t)$ - сила упругого взаимодействия между шайбой и стержнем, зависимость которой от времени обусловлена деформацией стержня под воздействием вибрации. Рассматривалось кулоновское трение, сила которого не превосходит по абсолютной величине $|f N(t)|$ и компенсирует другие воздействия в случае, если шайба покоится (в данной модели - силу инерции). 
Как было показано в публикации [15], принимая во внимание начальную деформацию или «натяг», с которым шайба насажена на стержень, можно переписать уравнение (20) в следующей форме:

$$
F_{f}= \begin{cases}-\eta f S_{c} E\left(\varepsilon_{n}^{0}-\frac{\rho A \omega^{2}(l-x)}{2 E} \sin \omega t\right), & \dot{x}>0 \\ -m A \omega^{2} \sin \omega t, \dot{x} \equiv 0 & \\ \eta f S_{c} E\left(\varepsilon_{n}^{0}-\frac{\rho A \omega^{2}(l-x)}{2 E} \sin \omega t\right), & \dot{x}<0\end{cases}
$$

Здесь $S_{c}$ - площадь области контакта стержень-шайба, а $E$ - модуль Юнга материала стержня. Следует потребовать также выполнения следующего условия для начальной деформации $\varepsilon^{0}$ :

$$
\varepsilon^{0} \geq \frac{\rho A \omega^{2} l}{2 E}
$$

В противном случае стержень и шайба могут потерять контакт, а поведение шайбы, насаженной на стердень с зазором может существенно отличаться от поведения, описываемого исходными уравнениями.

Микропроскальзывание и вибрационное перемещение в такой системе могут быть исследованы с использованием методов, представленных в книге [5] для исследования задачи о вибрационном перемещении тела на наклонной вибрирующей шероховатой плоскости.

Ориентируясь на применение метода поэтапного интегрирования, удобно разбить ось времени на интервалы так, что шайба, помещенная на стержень в состоянии относительного покоя, в каждый интервал начнет скольжение в «положительном» направлении - интервал $I_{+}$, останется в состоянии относительного покоя - интервал $I_{0}$, или начнет скольжение в «отрицательном» направлении - интервал $I_{-}$.

Движение в положительном направлении начинается при выполнении следующих условий:

$$
\begin{aligned}
& \sin \omega t>z_{+} \\
& z_{+}=\frac{2 \eta f S_{c} E \varepsilon_{n}^{0}}{A \omega^{2}\left(2 m+\eta f \rho S_{c}(l-x)\right)}
\end{aligned}
$$

«Отрицательный» интервал описывается условиями:

$$
\begin{aligned}
& \sin \omega t<z_{-} \\
& z_{-}=-\frac{2 \eta f S_{c} E \varepsilon_{n}^{0}}{A \omega^{2}\left(2 m-\eta f \rho S_{c}(l-x)\right)}
\end{aligned}
$$

Шайба остается в состоянии относительного покоя при выполнении следующих условий:

$$
\left\{\begin{array}{l}
\sin \omega t-z_{+}<0 \\
\sin \omega t-z_{-}>0
\end{array}\right.
$$

Движение шайбы рассматривается как множество фаз движения в «положительном» и «отрицательном направлении», а также фаз относительного покоя. Следует отметить, что нет четкого однозначного соответствия между фактическими фазами скольжения и упомянутыми выше интервалами $I$. Поведение шайбы во время фаз движения может быть описано следующими выражениями:

$$
\dot{x}(t)=\mp \frac{f S_{c} E \varepsilon_{n}^{0}}{m}\left(t-t^{*}\right)-A \omega\left(1 \pm \frac{f S_{c} \rho(l-x)}{2 m}\right)\left(\cos \omega t-\cos \omega t^{*}\right)+\dot{x}^{*}
$$




$$
\begin{aligned}
x(t)= & \mp \frac{f S_{c} E \varepsilon_{n}^{0}}{2 m}\left(t-t^{*}\right)^{2}+A \omega\left(1 \pm \frac{f S_{c} \rho(l-x)}{2 m}\right) \cos \omega t^{*}\left(t-t^{*}\right)- \\
& -A\left(1 \pm \frac{f S_{c} \rho(l-x)}{2 m}\right)\left(\sin \omega t-\sin \omega t^{*}\right)+\dot{x}^{*}\left(t-t^{*}\right)
\end{aligned}
$$

Верхние знаки - для положительной, нижние - для отрицательной начальной скорости соответственно. После остановки $(\dot{x}(t)=0)$ шайба переходит в фазу, соответствующую типу интервала, в который попадает $t$ - момент остановки: $I_{ \pm}$или $I_{0}$.

Программное обеспечение, разработанное на основе описанного выше метода, может быть применено для оценки рассеяния энергии или мощности диссипативных сил, которая входит как параметр в выражения для оценки износа в соединениях. Как показано ниже, износ пропорционален этой мощности. Диаграмма, показывающая зависимость мощности диссипативных сил в зависимости от амплитуды колебаний основания стержня на рис. 8 .

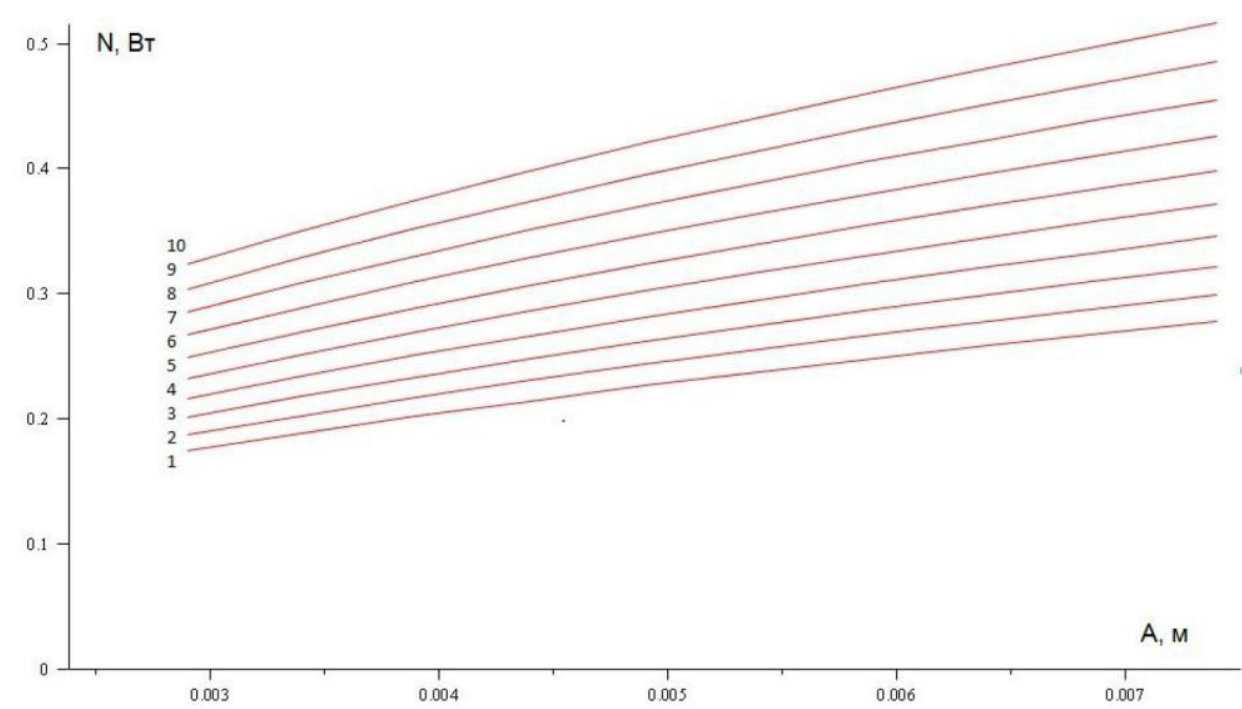

Рис. 8. Мощность диссипации в зависимости от амплитуды колебаний основания стержня для разных значений частоты колебаний (коэффициент перегрузки при этом меняется от 10 - кривая 1 до 12 - кривая 10)

\section{5. О некоторых других моделях.}

В числе других моделей, предложенных вне связи с проблемой износа, сошлемся на модели с упругими телами, изученные в работах $[6,9,12,13]$. Упругость контактирующих тел учитывается также в теории конструкционного гистерезиса [14]. При необходимости упомянутые модели могут также быть использованы для оценки износа при вибрации.

\section{6. Об износе номинально неподвижных соединений при вибрации и ударах.}

Энергия, затрачиваемая при проскальзывании тел за один период вибрации $T=2 \pi / \omega$, составляет $E_{1}=4 f N B$, а расход энергии за одну секунду, т.е. соответствующая мощность

$$
P=E_{1} / T=\frac{2}{\pi} f N B \omega .
$$

Будем исходить из предположения, что энергия, расходуемая на преодоление сил трения при взаимном проскальзывании контактирующих деталей, затрачивается на разрушение материалов этих деталей. Иными словами, будем считать, что скорость массового износа $W($ кг $/$ c), т.е. износ материала за одну секунду, пропорциональна мощности $P$ :

$$
W=\kappa P=\frac{2 \kappa}{\pi} f N B \omega
$$


Здесь коэффициент $\kappa$, имеющий размерность $\frac{\kappa 2}{c} \frac{c}{H м}=\frac{\kappa 2}{H} \frac{1}{M}=\frac{c^{2}}{M^{2}}$, представляет собой массовый износ, соответствующий затрате энергии $P=1$ Вт. Такое предположение согласуется с так называемой энергетической теорией износа [16-18], а также с данными практики измельчения твердых материалов (см. ниже) и данными об износе мелющих тел в барабанных мельницах ([19], с. 302).

Если обозначить через $F$ площадь контактирующих поверхностей, а через $\Delta$ величину скорости линейного износа, то будет

$$
W=\rho F \Delta, \quad \Delta=\frac{W}{\rho F}=\frac{2 \kappa f \sigma B \omega}{\pi \rho}(\mathrm{м} / \mathrm{c}),
$$

где $\sigma=N / F$ - нормальное давление между контактирующими деталями. Зная значение скорости линейного износа $\Delta$, нетрудно оценить время $T_{*}$, за которое износ достигнет критического значения $\delta$ :

$$
T_{*}=\delta / \Delta(\mathrm{c})=\delta / 3600 \cdot \Delta(\mathrm{ч})
$$

Величину $\kappa$ в формулах (20), (21) можно рассматривать как эмпирически определяемый коэффициент; данных о величине этого коэффициента нам обнаружить не удалось. Поэтому попытаемся хотя бы грубо оценить этот коэффициент на основе косвенных данных.

В теории дробления и измельчения $[19,20]$ хорошо известна величина $\varphi(D, d)$, представляющая собой затрату энергии, необходимую для разрушения килограмма материала от некоторой средней крупности $D$ до средней крупности $d$. Очевидно, что эта величина обратна введенному выше коэффициенту $\kappa$, т.е.

$$
\varphi(D, d)=1 / \kappa \quad\left(B m \cdot c / \kappa 2=\mathcal{M}^{2} / c^{2}\right)
$$

По результатам работ $[21,22]$ продукты износа рассмотренных пар материалов имели размеры 40-50 мкм. В измельчительных аппаратах типа дисковых истирателей при измельчении руды до такой крупности по весьма приближенным оценкам затрачивается энергия $\varphi$ порядка 2000 кВт•ч/т $=7.2 \cdot 10^{6}$ Вт сс/кг. Учитывая, что для такого же измельчения металлов и при таком «несовершенном» способе измельчения как фрикционный износ, энергозатраты значительно выше, примем в приводимом ниже примере расчета сугубо ориентировочно коэффициент $\varphi$ на два порядка большим, т.е. $\varphi \approx 10^{9} \mathrm{~B}$ т с $/$ кг.

Пусть $\rho=7.8 \Gamma / \mathrm{cm}^{3}=7.8 \cdot 10^{3}$ кг $/ \mathrm{m}^{3}, f=0.3, \sigma=10 \mathrm{H} / \mathrm{мм}^{2}=10^{7} \mathrm{H} / \mathrm{M}^{2}, \omega=314 \mathrm{c}^{-1}, \mathrm{~B}$ $=1$ мкм $=10^{-6}$ м. Тогда согласно формулам (21) и (22) получим $\Delta=\frac{2 \cdot 0.3 \cdot 10^{7} \cdot 10^{-6} \cdot 314}{3.14 \cdot 10^{9} \cdot 7.8 \cdot 10^{3}}=0.769 \cdot 10^{-10} \mathrm{M} / \mathrm{c} \quad$ и $\quad$ при $\quad \delta=1 \mathrm{мм}=10^{-3} \mathrm{M} \quad$ находим $T_{*}=\delta / \Delta=\frac{10^{-3}}{0.769 \cdot 10^{-10}}=1.3 \cdot 10^{7} \mathrm{c}=\frac{1.3 \cdot 10^{6}}{3600 \cdot 24}=150$ суток. Если же принять полуразмах скольжения $B=0.1 \mathrm{мм}=10^{-4}$ м, то время $T_{*}$ уменьшится в 100 раз, т.е. составит всего 1.5 суток.

Отметим, что в ряде случаев проскальзывание деталей под действием ударов и вибрации удается уменьшить или даже устранить путем конструктивных или технологических решений. Примерами могут служить решения по снижению конструкционного гистерезиса, рассмотренные в книгах $[14,23]$, а также патент [24].

\section{7. Некоторые рекомендации по конструированию и эксплуатации машин, работающих в условиях вибрации и ударов}

1). В конструкциях, работающих в условиях удара и вибрации, следует с большой осторожностью рассчитывать на взаимную неподвижность деталей, контактирующих посредством сил сухого трения. Это относится, в частности, к болтовым фланцевым соединениям шаровых и стержневых мельниц, дробилок, грохотов, а также энергетических машин. 
2). В результате микропроскальзывания контактирующих деталей под действием ударов и вибраций происходит их износ; возможно также одностороннее смещение деталей (пример - самоотвинчивание резьбовых соединений). Это может при плохом обслуживании привести к аварийным ситуациям и даже к катастрофам.

3). Если при действии вибрации можно исключить взаимное проскальзывание контактирующих деталей посредством достаточно большого натяга, то в случае ударных нагрузок гарантировать микропроскальзывание нельзя даже при наличии весьма значительного натяга.

4). Проскальзывание деталей под действием вибрации и ударов в ряде случаев можно уменьшить и даже устранить путем надлежащих конструктивных и технологических решений.

5). При назначении периодичности обслуживания рассматриваемых деталей и их соединений необходимо учитывать, что согласно предложенной теории скорость износа увеличивается «сильнее», чем пропорционально амплитуде и в еще большей мере - с увеличением частоты колебаний.

\section{8. Заключение.}

В настоящей работе рассмотрено действие вибрации и ударов на номинально взаимно неподвижные детали, например, болтовые и фланцевые соединения. При действии вибрации и ударов такие детали обнаруживают взаимную микроподвижность и, как следствие, подвержены износу. На уровне двух моделей получены формулы для скорости износа, содержащие один эмпирический коэффициент. По физическому смыслу этот коэффициент представляет собой износ на единицу энергии, затрачиваемой при взаимном проскальзывании деталей. На основе полученных результатов даны некоторые рекомендации по конструированию и ремонтно-техническому обслуживанию соответствующих машин.

\section{Литература}

1. Zhu M.H., Zhou Z.R. On the mechanisms of various fretting wear modes // Tribology International. 2011. Vol. 44. P. 1378-1388.

2. Yong Liu, Jin-Quan Xu, Yoshiharu Mutoh. Evaluation of fretting wear based on the frictional work and cyclic saturation concepts // International Journal of Mechanical Sciences. 2008. Vol. 50. P. 897-904.

3. Helmi Attia. A generalized fretting wear theory // Tribology International. 2009. Vol. 42. P. 1380-1388.

4. Перфильева Н.В Динамическая модель механического контактирования условнонеподвижных соединений. Автореф. дисс. ... докт. техн. наук. - Томск, 2003. - 39 с. $410 \mathrm{c}$.

5. Блехман И.И., Джанелидзе Г.Ю. Вибрационное перемещение. - М.: Наука, 1964. -

6. Блехман И.И. Вибрационная механика. - М.: Физматлит, 1994, 400 с. (англ. перевод: Blekhman I.I. Vibrational Mechanics. (Nonlinear Dynamic Effects, General Approach, Applications). - Singapore et al: - World Scientific Publishing Co., 2000. - 510 p.)

7. Толстой Д.М. Собственные колебания ползуна, зависящие от контактной жесткости, и их влияние на трение // ДАН СССР. 1963. Т.153, № 4. С. 820-824.

8. Толстой Д.М., Каплан Р.Л. К вопросу о фрикционных автоколебаниях и скоростной зависимости силы трения // Теория трения и износа. - М.: Наука, 1965. С. 44-49.

9. Рыспеков К.М. Исследование эксплуатационной надежности и совершенствование ремонтного обслуживания измельчительного оборудования (на примере обогатительной фабрики Джезказганского ГМК): Автореф. дис. ... канд. техн. наук. - Л., 1982.

10. Лойцянский Л.Г., Лурье А.И. Теоретическая механика. Ч. ІІІ. - Л.-М.: ОНТИ, 1934. $624 \mathrm{c}$.

11. Якимова К.С. Вибрационное перемещение двухмассной колебательной системы // Изв. Акад. наук СССР, МТТ. 1969, № 5. С.20-30. 
12. Флорина Н.В. Штамп на упругом основании под действием переменных нагрузок при наличии проскальзывания: Автореф. дисс. ... канд. техн. наук. - Л., 1964.

13. Никитин Л.В. Статика и динамика твердых тел с внешним сухим трением. - М.: Московский лицей, 1998. - 272 с.

14. Пановко Я.Г. Механика деформируемого твердого тела. - М.: Наука, 1985. - 288 с.

15. Blekhman I.I., Ivanov K.S. On a Chelomey pendulum problem // Proc. of the XXXVII Summer School "Advanced Problems in Mechanics" (APM - 2009). St. Petersburg (Repino), June 30-July 5, 2009. - St. Petersburg, IPME RAS, 2009. P. 112-117.

16. Фляйшер Г. Энергетический метод определения износа / Исследования по триботехнике. М.: НИИ информации по машиностроению, 1975. С. 277-291.

17. Крагельский И.В., Добычин М.Н., Комбалов В.С. Основы расчетов на трение и износ. М.: Машиностроение, 1977. - 526 с.

18. Ибатуллин И.Д. Кинетика усталостной повреждаемости и разрушения поверхностных слоев. - Самара: Самар. гос. техн. ун-т, 2008. -387 с. (URL: http://www.tm.samgtu.ru/sites/tm.samgtu.ru/files/kinetika.pdf).

19. Андреев С.Е., Перов В.А, Зверевич В.В. Дробление, измельчение и грохочение полезных ископаемых. Изд. 3-е. М.: Недра, 1980. - 415 с.

20. Справочник по обогащению руд. Т. 1 - М.: Недра, 1972. - 447 с.

21. Фадин Ю.А., Полевая О.В., Попов И.М. Размеры и форма частиц при сухом трении металлов // Письма в ЖТФ. 1994. Т. 20, вып. 17. С. 47-51.

22. Фадин Ю.А. Кинетика разрушения поверхности металлических материалов при трении. Автореф. дисс. ... докт. техн. наук. - Л., 1997. - 30 с.

23. Блехман И.И. Что может вибрация? О вибрационной механике и вибрационной технике. - М.: Наука, 1998. 208 с.

24. Патент 2256108 РФ. Способ изготовления болтового соединения. / Ж.М. Бледнова, Н.А. Махутов, М.И. Чаевский. - Опубл. 10.07.2005 г.

Поступила: 01.10.12. 